\title{
ARTICLE
}

Cite this: DOI: $10.1039 /$ xoxxooooox

Received ooth January 2012, Accepted ooth January 2012

DOI: $10.1039 / \times 0 \times x 00000 x$

www.rsc.org/

\section{Stereoselective innovative synthesis and biological evaluation of new real carba analogues of minimal epitope Man $\alpha(1,2)$ Man as DC-SIGN inhibitors}

\author{
Vittorio Bordoni, ${ }^{a}$ Vanessa Porkolab, ${ }^{b}$ Sara Sattin, ${ }^{c}$ Michel Thépaut, ${ }^{b}$ Ileana Frau, ${ }^{a}$ \\ Lucilla Favero, ${ }^{a}$ Paolo Crotti, ${ }^{a}$ Anna Bernardi, ${ }^{c}$ Franck Fieschi, ${ }^{b}$ Valeria Di \\ Bussolo $^{* d}$
}

\begin{abstract}
Antagonists of the C-type lectin DC-SIGN are promising therapeutic agents against viruses and bacteria. The development of glycomimetics ligands for DC-SIGN has so far proved to be challenging, since this membrane-protein presents four carbohydrate-binding domains (CRD) that specifically recognize mannose and fucose. In the recent past, we were able to develop inhibitors mimicking the minimal natural epitope Man $\alpha(1,2)$ Man using a mannoside with conformationally restricted dimethyl cycloexandicarboxylate-based aglycons designed to exploit the high enzymatic stability and to generate multivalent or solid supported systems as potent lectin ligands. Herein we describe the innovative synthesis of a different class of pseudodisaccharides, mimic of the natural $\operatorname{Man} \alpha(1,2)$ Man moiety, characterized by the presence of a real D-carbamannose unit instead of a simpler mimic structure. Their chemical synthesis and biological activity using an SPR inhibition assay are reported. These pseudodisaccharides display inhibition values similar to those of the natural disaccharide Mano(1,2)Man, with a good affinity for DC-SIGN and can be considered as possible candidates for further structural modifications towards improved inhibitors.
\end{abstract}

\section{Introduction}

A broad range of biological processes including cellular adhesion, signaling, migration and infection are mediated by carbohydrate-lectin interactions. $^{1-4}$ In this framework, DCSIGN (Dendritic Cell-Specific Intercellular adhesion molecule3 (ICAM-3)-Grabbing Non-integrin) is a C-type lectin involved in the recognition of viruses and pathogens at the mucosal level. In particular the interaction between gp120 on the surface of HIV-1 and DC-SIGN allows the delivery of the virus to tissues rich in $\mathrm{CD}^{+} \mathrm{T}$ cells, such as lymphoid organs, playing therefore a crucial role in the infection process at the mucosal level. ${ }^{5,6}$ The main carbohydrate ligand present on gp120 and recognized by the DC-SIGN carbohydrate-binding domains (CRDs) is the high mannose glycan Man $_{9}($ GlcNAc) 2 (Man9, 1, Figure 1). Therefore, derived glycomimetic structures can inhibit DC-SIGN action and are of high interest against HIV infections as antiviral agents. ${ }^{7-17}$ Notably, the 1,2-mannobioside disaccharide unit, Man $(1,2)$ Man 2 (Figure 1), contained in all three arms D1, D2 and D3 of Man9, is one of the minimal epitopes for binding to DC-SIGN. To underline the efficiency of this epitope and the importance of polyvalent systems, the incorporation of the natural 1,2-mannobioside into gold glyconanoparticles (Au-GNPs) increases the inhibitory activity by more than four orders of magnitude, compared to that of the monovalent disaccharide. ${ }^{18}$ We previously reported that disaccharide mimetics 3a-c (Figure 1) show three dimensional structure and conformational behavior comparable to those of Mano(1,2)Man 2 and display a micromolar activity against DC-SIGN mediated Ebola viral infection, to demonstrate for the first time that DC-SIGN antagonism by small molecules might be used to inhibit viral transfection mediated by DCSIGN. ${ }^{10,17,19}$

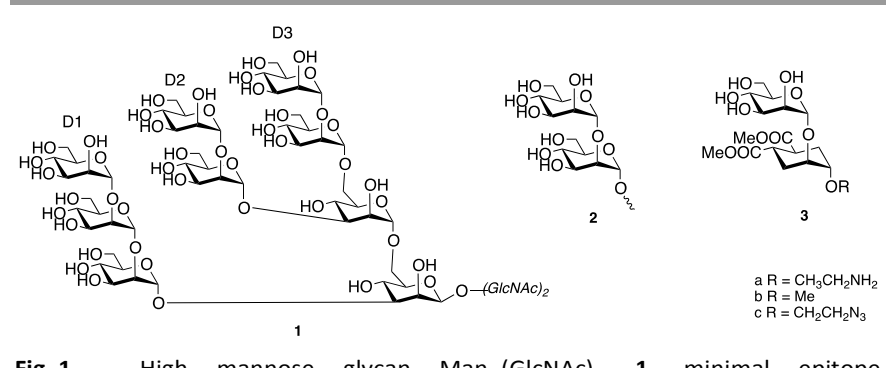

Mana(1,2)Man 2 and chemical structure of disaccharide mimetics 3a-c.

Herein, we describe the innovative synthesis of pseudodisaccharides 4 and 5 (Figure 2), real carba analogues of the natural disaccharide epitope 2, and their biological evaluation as prospective DC-SIGN ligands. In our approach, the non reducing portion of the natural disaccharide 
Mano(1,2)Man is replaced by a carbasugar, in view of an improved drug-like character of the resulting glycomimetic compared to the natural carbohydrate. Indeed, sugar mimics are generally more soluble and membrane penetrant, less hydrophilic and less metabolically labile than the sugars themselves. $^{20}$
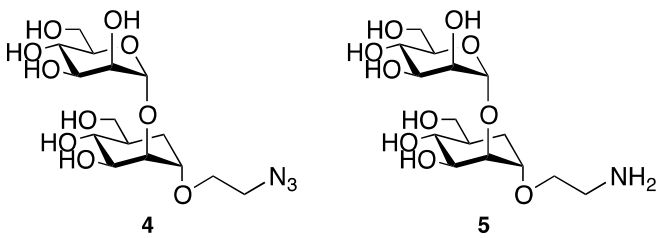

Fig. 2 Chemical structures of pseudodisaccharides $\mathbf{4}$ and $\mathbf{5}$ real carba analogues of epitope 2.

\section{Results and discussion}

\section{Synthesis of pseudodisaccharides 4 and 5.}

The stereoselective synthesis of pseudodisaccharides $\mathbf{4}$ and $\mathbf{5}$ proceeds through the construction of skeleton $\mathbf{8}$ (Scheme 1) by glycosylation of the new carbamannose glycosyl acceptor 7 with tetrabenzoyl mannose trichloroacetimidate $\mathbf{6}$ as typical glycosyl donor. ${ }^{21}$

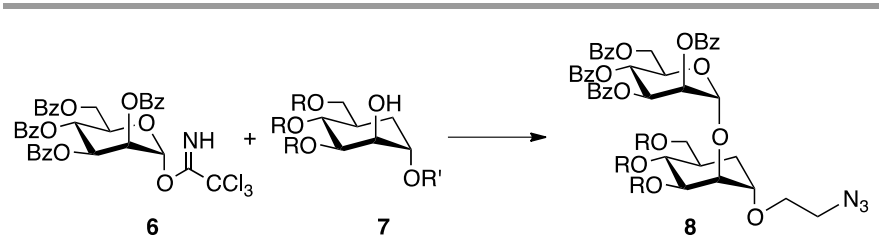

Scheme 1 Stereoselective synthesis of skeleton $\mathbf{8}$.

Two carbamannose units $\mathbf{1 5}$ and 16, carrying either acetyl or benzyl protecting groups, were prepared and studied in this reaction. Both were prepared starting from the pivotal carba epoxy diol 11 obtained from $m$-CPBA epoxidation of cyclohexene $\mathbf{1 0}$ in turn prepared from tri-O-acetyl-D-glucal $\mathbf{9},{ }^{22}$ as shown in Scheme 2.

The allyl alcohol $\mathbf{1 0}$ is epoxidized stereoselectively by MCPBA, which reacts with the double bond face in syn to the allylic hydroxy group. ${ }^{23,24}$ Key epoxide $\mathbf{1 1}$ was then separately elaborated to give tri-O-benzyl-5a-carba- $\beta$-epoxide (+)$14^{25}$ upon treatment with $\mathrm{NaH} / \mathrm{BnBr}$ in $\mathrm{DMF}$, or debenzylated at $\mathrm{C}(6)$, under reductive conditions with $\mathrm{H}_{2} /(10 \%) \mathrm{Pd}-\mathrm{C}$, to afford $\beta$-epoxy triol 12 which was in situ acetylated $\left(\mathrm{Ac}_{2} \mathrm{O} / \mathrm{Py}\right)$, to give tri- $O$-acetyl-5a-carba- $\beta$-epoxide $(+)-\mathbf{1 3}^{25}$ (Scheme 2). Epoxides (+)-13 and (+)-14 represent the carbaglycosylating agents which were subjected to ring opening reaction with freshly prepared 1.3M 2-azido-1-ethanol in $\mathrm{CH}_{2} \mathrm{Cl}_{2}{ }^{26}$ in the presence of a catalytic amount of $\mathrm{Cu}(\mathrm{OTf})_{2}$ as the Lewis acid catalyst. ${ }^{27}$ In accordance with reports by Ogawa and Cumpstey ${ }^{28,29}$ on the behavior of 1,2-epoxides with $\beta$-manno configuration like $(+)-13$ and $(+)-14$, nucleophilic attack exclusively occurs in a trans-diaxial fashion, at the less sterically hindered and more electronically favored oxirane $\mathrm{C}(1)$ carbon. This way, the corresponding 2-azido-1-ethoxycyclohexanol derivatives $(+)-15$ from $(+)-13$,

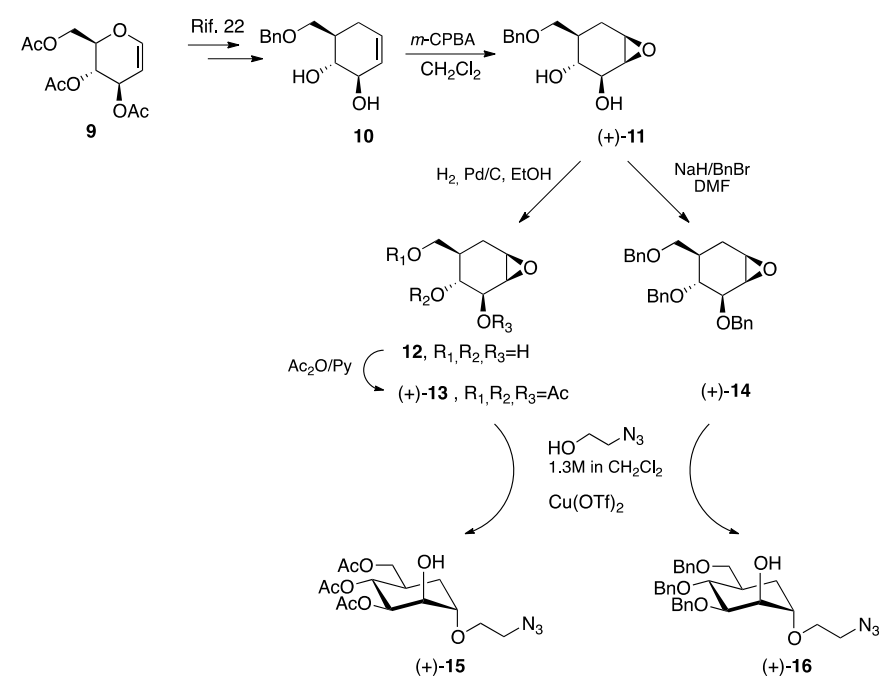

Scheme 2 Stereoselective synthesis of carbamannose glycosyl acceptor 15 and 16.

and (+)-16 from $(+)-\mathbf{1 4}$, are obtained through a completely regio- and stereoselective ring opening process.

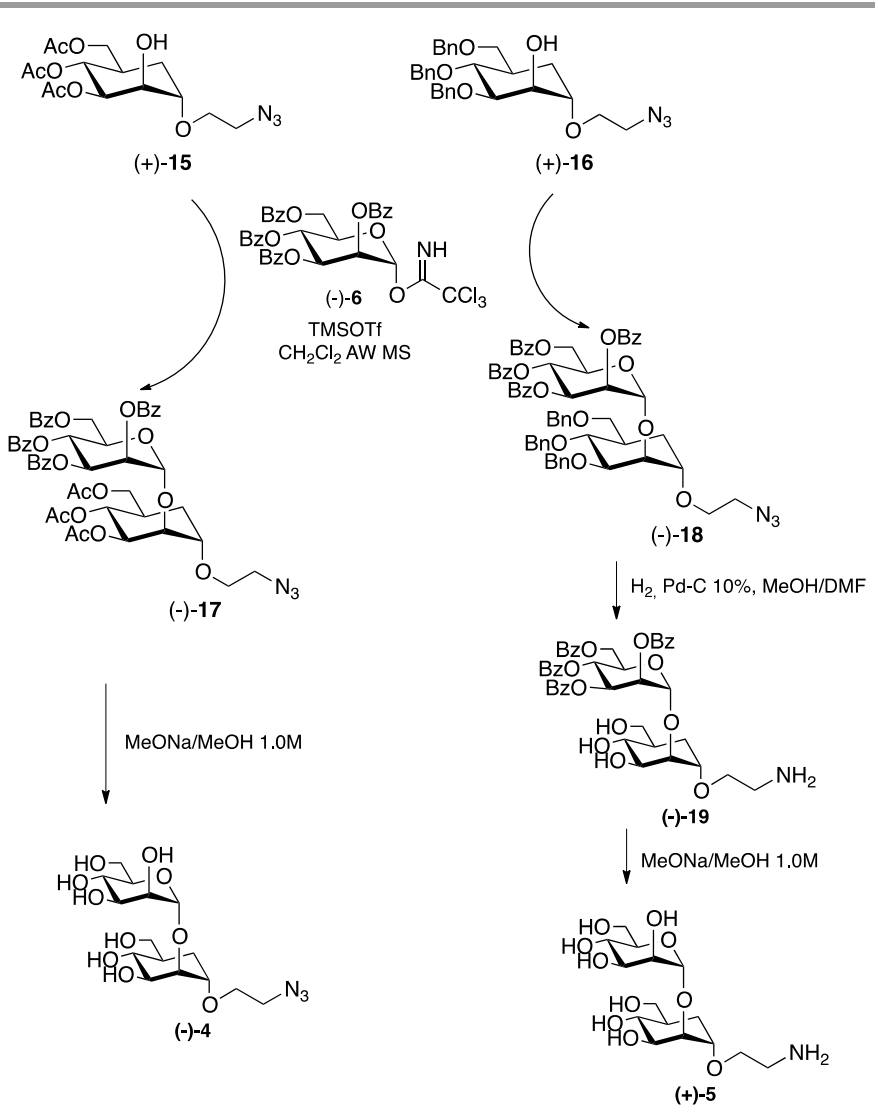

Scheme 3 Stereoselective synthesis of glycoconjugates (-)-4 and (+)-5.

Glycosylation reaction of glycosyl acceptors (+)-15 and (+)-16 by the typical mannosyl donor $\mathbf{6}$ was successfully carried out in standard conditions (TMSOTf in $\mathrm{CH}_{2} \mathrm{Cl}_{2},-20^{\circ} \mathrm{C}, 2 \mathrm{~h}$ ), to afford glycoconjugates (-)-17 and (-)-18 in a completely $\alpha-$ 
stereoselective fashion. Catalytic hydrogenolysis of (-)-18 reduced the azido functionality and removed the benzyl protecting groups to afford pseudodisaccharide (-)-19. Subsequent Zemplén deprotection provided the final pseudodisaccharide (+)-5 with a $\alpha$-aminoethoxy chain at pseudoanomeric C(1) in 69\% yield after recrystallization from $i-\operatorname{Pr}_{2} \mathrm{O}$. On the other hand, Zemplén deprotection of glycoconjugate (-)-17, afforded the $\alpha$-azidoethoxy pseudodisaccharide (-)-4 in $88 \%$ yield (Scheme 3 ).

Preparation of pseudodisaccharides (-)-4 and (+)-5 represents the first example of stereoselective synthesis of real carba analogues of minimal natural epitope Man $\alpha(1,2)$ Man thus, their biological activity as DC-SIGN antagonists was evaluated using an inhibition assay based on Surface Plasmon Resonance (SPR) technique.

\section{DC-SIGN inhibition assays}

The affinity of the newly synthesized sugar conjugates $\mathbf{4}$ and $\mathbf{5}$ for DC-SIGN was tested by competition experiments using surface plasmon resonance (SPR), as previously described. ${ }^{11} \mathrm{~A}$ CM4 sensorchip was functionalized with mannosylated Bovine Serum Albumine (BSA-Mannotriose) and a fixed amount of the extracellular domain of DC-SIGN was injected over the surface with increasing concentrations of ligands. The natural 1,2mannobioside $\mathbf{2}$ and its mimic $\mathbf{3}$ were used as control. From the inhibition curves (Fig. 3) and according to 4-parameter model, an $\mathrm{IC}_{50}$ of about $1 \mathrm{mM}$ was estimated for all the compounds.

These data show that the new pseudodisaccharides (+)-5 and ()-4 have inhibitory potency similar to the natural disaccharide Mano1-2Man,. The $\mathrm{IC}_{50}$, extracted from inhibition curves are $964 \pm 14.6 \mu \mathrm{M}$ for $2,941 \pm 20.2 \mu \mathrm{M}$ for $3,1467 \pm 32.1 \mu \mathrm{M}$ for 4 and $1453 \pm 30.6 \mu \mathrm{M}$ for 5 . Thus, taking into account their enzymatic stability, (+)-5 and (-)-4 could be considered as possible candidates to prepare multivalent systems to be used as inhibitors of viral infection.
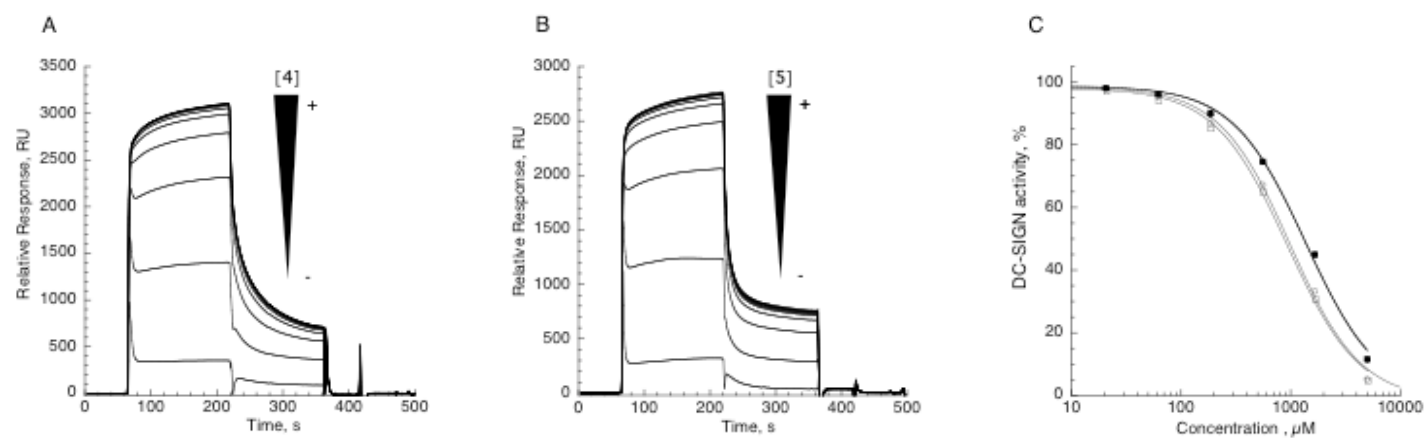

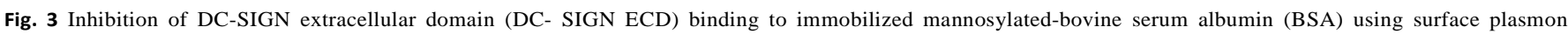

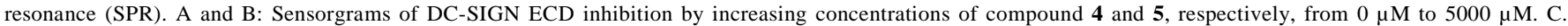
Comparison of the inhibitory potency of $\mathbf{2}(\mathrm{O}), \mathbf{3}(\square), \mathbf{4}(\bullet)$, and $\mathbf{5}(\boldsymbol{\square})$ toward the DC-SIGN ECD mannosylated-BSA interaction determined by competition assays.

\section{Conclusions}

We have successfully planned and achieved the innovative stereospecific synthesis of the two glycoconjugates (-)-4 and $(+)-5$, which were intended to represent mimics of the natural Mano(1,2)Man moiety, characterized by the presence of a real D-carbamannose unit. These glycoconjugates were designed to mime the minimal natural epitope $\operatorname{Man} \alpha(1,2)$ Man of the high mannose glycan $\operatorname{Man}_{9}(\text { GlcNAc })_{2}$, which is principal carbohydrate ligand recognized by the DC-SIGN. The biological assays evaluated using an inhibition (binding affinity?) assay based on Surface Plasmon Resonance (SPR) technique, displayed a remarkable activity of new pseudodisaccharides (-)-4 and (+)-5 having inhibitory potency similar to the natural disaccharide Mana1-2Man. These results confirm the validity of the DC-SIGN antagonism by small molecules and pave the way to more extensive studies of enzymatically stable glycoconjugates that are useful to prepare efficient multivalent systems as DC-SIGN inhibitors.

\section{Experimental}

\section{Materials and methods}

All solvents and chemicals were used as purchased without further purification. Chromatographic separations were performed on silica gel columns by flash (Kieselgel 40, 0.040$0.063 \mathrm{~mm}$; Merck) or by automated chromatography with Isolera ${ }^{\circledR}$ Biotage. Reactions were followed by thin-layer chromatography (TLC) on Merck aluminum silica gel $\left(60 \mathrm{~F}_{254}\right)$ sheets that were visualized under a UV lamp. Evaporation was performed in vacuo (rotating evaporator). Sodium sulfate was always used as the drying agent. Melting points were determined with a Kofler hot-stage apparatus and are uncorrected. Optical rotations were measured with a ATAGO AP-300 Automatic Polarimeter at $25^{\circ} \mathrm{C}$. Proton $\left({ }^{1} \mathrm{H}\right)$ and carbon $\left({ }^{13} \mathrm{C}\right)$ NMR spectra were obtained with a Bruker Avance 250 $\mathrm{MHz}$ spectrometer using the indicated deuterated solvents. Chemical shifts are given in parts per million (ppm) ( $\delta$ relative to residual solvent peak for ${ }^{1} \mathrm{H}$ and ${ }^{13} \mathrm{C}$ ). FTIR spectra were obtained with a IR Cary 600 FTIR (Agilent Tecnologies).Yields refer to isolated and purified products. High-resolution mass spectrometry (HRMS) analysis were performed using a Waters Xevo ${ }^{\circledR}$ G2-S QTof at Mass Spectrometry facility, PCN-ICMG of Grenoble. Compounds are dissolved in 1:1 acetonitrile/water (concentration 10E-5 M). 


\section{Synthetic procedures}

6-O-Benzyl-1,2-anhydro-5a-carba- $\beta$-D-mannopyranose (11). MCPBA (0.627 g, $3.64 \mathrm{mmol}, 1.2$ equiv) was added to a solution of trans diol $\mathbf{1 0}^{23}$ (0.709 g, $3.03 \mathrm{~mol}, 1.0$ equiv) in anhydrous $\mathrm{CH}_{2} \mathrm{Cl}_{2}(17 \mathrm{~mL})$ at $0^{\circ} \mathrm{C}$ and the reaction mixture was stirred at room temperature. After 18h, the reaction was diluted with $\mathrm{CH}_{2} \mathrm{Cl}_{2}$, the organic phase was washed with $10 \%$ aqueous $\mathrm{Na}_{2} \mathrm{~S}_{2} \mathrm{O}_{3}$, saturated aqueous $\mathrm{NaHCO}_{3}$ and brine, dried $\left(\mathrm{Na}_{2} \mathrm{SO}_{4}\right)$ and concentrated to afford a crude reaction product (0.585 g), which was subjected to a flash chromatography. Elution with 3:7 hexane/AcOEt mixture afforded $\boldsymbol{\beta}$ epoxide 11 (0.438 g, 59\% yield), pure as a white solid: $\mathrm{mp} 86-88{ }^{\circ} \mathrm{C} . \mathrm{R}_{\mathrm{f}}=$ 0.13 (3:7 hexane/AcOEt). $[\alpha]^{20}{ }_{\mathrm{D}}+8.03\left(c 0.61, \mathrm{CHCl}_{3}\right)$. FTIR (neat) $v$ 3360.2, 3255.3, 3026.3, 2920.5, 2852.6, 1453.5, 1362.3, 1257.7, 1112.6, 1057.9, 1015.3, 933.3, 731.7, 695.4 $\mathrm{cm}^{-1} .{ }^{1} \mathrm{H}$ NMR (250 MHz; $\mathrm{CDCl}_{3}$ ) $\delta 7.20-7.43$ (m, 5H; Ar), 4.54 (d, $J=12.0 \mathrm{~Hz}, 1 \mathrm{H}$; benzylic), 4.48 (d, $J=12.0 \mathrm{~Hz}, 1 \mathrm{H}$; benzylic), 3.84 (dd, $J=8.2,1.7 \mathrm{~Hz}, 1 \mathrm{H} ; \mathrm{H}_{3}$ ), 3.61 (dd, $J=10.2$, $8.3 \mathrm{~Hz}, 1 \mathrm{H} ; \mathrm{H}_{4}$ ), 3.52 (dd, $J=9.2,4.8 \mathrm{~Hz}, 1 \mathrm{H} ; \mathrm{H}_{6}$ ), 3.47 (dd, $J$ = 9.2, $4.8 \mathrm{~Hz}, 1 \mathrm{H} ; \mathrm{H}_{6}$ ), 3.37 (ddd, $J=4.0,1.7,0.6 \mathrm{~Hz}, 1 \mathrm{H}$; $\mathrm{H}_{2}$ ), 3.27 (t, $\left.J=4.3 \mathrm{~Hz}, 1 \mathrm{H} ; \mathrm{H}_{1}\right), 2.00-2.12\left(\mathrm{~m}, 1 \mathrm{H} ; \mathrm{H}_{5 \mathrm{a \alpha}}\right.$ ), $1.79-1.95$ (m, $1 \mathrm{H} ; \mathrm{H}_{5}$ ), 1.73 (dd, $\left.J=14.2,11.2 \mathrm{~Hz}, 1 \mathrm{H} ; \mathrm{H}_{5 \mathrm{a} \beta}\right)$. ${ }^{13} \mathrm{C} \mathrm{NMR}\left(75 \mathrm{MHz} ; \mathrm{CDCl}_{3}\right) \delta 137.84,128.63,127.99,127.78$, 74.64, 73.52, 73.11, 72.24, 56.64, 53.30, 38.90, 26.39.

\section{3,4,6-tri-O-benzyl-1,2-anhydro-5a-carba- $\beta$-D- mannopyranose (14). ${ }^{25}$}

A $60 \%$ dispersion of $\mathrm{NaH}(0.374 \mathrm{~g}, 9.36 \mathrm{mmol}, 4.0$ equiv) was added to a solution of $\boldsymbol{\beta}$ epoxide 11 (0.584 g, $2.34 \mathrm{mmol}, 1.0$ equiv) in anhydrous DMF $(8 \mathrm{~mL})$ at $0^{\circ} \mathrm{C}$ and the reaction mixture was stirred at the same temperature for 30 minutes and then at room temperature for 30 minutes. After cooling at $0^{\circ} \mathrm{C}$, $\mathrm{BnBr}(0.70 \mathrm{~mL}, 5.86 \mathrm{mmol}, 2.5$ equiv) was added dropwise and the solution was stirred $2 \mathrm{~h}$ at room temperature. Dilution with $\mathrm{Et}_{2} \mathrm{O}$ and ice and evaporation of the washed (brine) and dried $\left(\mathrm{Na}_{2} \mathrm{SO}_{4}\right)$ organic solution afforded a crude reaction mixture (0.920 g), which was subjected to a flash chromatography. Elution with an 8:2 hexane/AcOEt mixture afforded tri- $O$ bernzyl $\boldsymbol{\beta}$ epoxide 14 (0.740 g, 73\% yield), pure as a white solid. mp 53-55 ${ }^{\circ} \mathrm{C} . \mathrm{R}_{\mathrm{f}}=0.24$ (8:2 hexane/AcOEt). $[\alpha]^{20}{ }_{\mathrm{D}}$ +32.7 (c 0.81, $\mathrm{CHCl}_{3}$ ). FTIR (neat) v 3025.0, 2913.9, 2852.0, $1946.5,1873.9,1744.0,1453.3,1366.2,1102.3,1081.5$, 1023.5, 930.9, 737.6, $695.5 \mathrm{~cm}^{-1} .{ }^{1} \mathrm{H}$ NMR (250 MHz, $\mathrm{CDCl}_{3}$ ) ઈ 7.27-7.45 (m, 14H; Ar), 7.20-7.25 (m, 1H; Ar), 4.84 (d, $J$ $=10.4 \mathrm{~Hz}, 1 \mathrm{H}$; benzylic), 4.82 (s, 2H; benzylic), 4.52 (d, J $=10.4 \mathrm{~Hz}, 1 \mathrm{H}$; benzylic), 4.46 (s, 2H; benzylic), 3.83 (dd, $J=$ 8.1, $1.9 \mathrm{~Hz}, 1 \mathrm{H}$; H3), 3.65 (dd, $J=10.9,8.1 \mathrm{~Hz}, 1 \mathrm{H}$; H4), 3.453.55 (m, 2H; H6 + H6'), 3.34 (dd, $J=4.1,1.8 \mathrm{~Hz}, 1 \mathrm{H} ; \mathrm{H} 2$ ), $3.26(\mathrm{t}, J=3.9 \mathrm{~Hz}, 1 \mathrm{H} ; \mathrm{H} 1), 1.97-2.20(\mathrm{~m}, 2 \mathrm{H} ;$; $\mathrm{H} 5 \mathrm{a} \alpha+$ H5aß), 1.90-1.63 (m, 1H; H5).

${ }^{13} \mathrm{C}$ NMR (75 MHz, $\mathrm{CDCl}_{3}$ ) $\delta 139.1,139.0,138.9,128.8$, 128.7, 128.5, 128.3, 128.1, 128.0, 127.9, 81.9, 78.2, 75.6, 73.5, 72.8, 70.5, 55.7, 53.9, 40.3, 27.4 .
(2-azidoethyl)-3,4,6-tri-O-benzyl-5a-carba- $\alpha-D-$ mannopyranoside (16).

Tri-O-bernzyl $\boldsymbol{\beta}$ epoxide 14 (0.370 g, $0.859 \mathrm{mmol}, 1$ equiv) was dissolved in a solution $1.3 \mathrm{M}$ 2-azido-1-ethanol in $\mathrm{CH}_{2} \mathrm{Cl}_{2}$ (2.65 $\mathrm{mL}, 3.44$ mmol, 4.0 equiv) freshly prepared ${ }^{25}$ and $\mathrm{Cu}(\mathrm{OTf})_{2}(0.124$ g, 0.344 mmol, 0.4 equiv) was added. The reaction mixture was stirred for $18 \mathrm{~h}$ at room temperature. Dilution with $\mathrm{CH}_{2} \mathrm{Cl}_{2}$ and evaporation of the washed (1:1 $\mathrm{NaHCO}_{3} / \mathrm{NH}_{4} \mathrm{Cl}$ mixture and brine) and dried $\left(\mathrm{Na}_{2} \mathrm{SO}_{4}\right)$ organic solution afforded a crude reaction mixture $(0.363 \mathrm{~g})$ which was subjected to a flash chromatography. Elution with 7:3 hexane/AcOEt mixture afforded product 16 (0.304 g, 68\% yield), pure as a pale yellow liquid. $\mathrm{R} f=0.50$ (7.3 hexane/AcOEt); $[\alpha]^{20}{ }_{\mathrm{D}}+22.34$ (c. $0.67, \mathrm{CHCl}_{3}$ ). FTIR (neat) $v$ 3449.6, 2927.2, 2871.8, 2103.9, 1737.6, 1284.4, 1242.6, 1109.5, 1048.3, 993.4, 731.3, $696.8 \mathrm{~cm}^{-1} .{ }^{1} \mathrm{H}$ NMR (250 $\left.\mathrm{MHz} ; \mathrm{CDCl}_{3}\right) \delta$ 7.20-7.40 (m, 15H; Ar), 4.83 (d, $J=10.9$ Hz, 1H; benzylic), 4.72 (d, $J=11.5 \mathrm{~Hz}, 1 \mathrm{H}$; benzylic), 4.66 (d, $J=11.5 \mathrm{~Hz}, 1 \mathrm{H}$; benzylic), 4.51 (d, $J=10.9 \mathrm{~Hz}, 1 \mathrm{H}$; benzylic), 4.47 (s, 2H; benzylic), 4.13 (t, $J=3.4$ $\mathrm{Hz}, 1 \mathrm{H}$; H1), 3.80 (dd, $J=9.0,3.0 \mathrm{~Hz}, 1 \mathrm{H}$; H2), 3.45-3.75 (m, 6H), 3.18-3.39 (m, 2H; ; H6 + H6'), 1.95-2.14 (m, 1H; ; H5), 1.85-1.92 (m, 2H; $\mathrm{H} 5 \mathrm{a} \alpha+\mathrm{H} 5 \mathrm{a} \beta)$.

${ }^{13} \mathrm{C}$ NMR (63 $\left.\mathrm{MHz}, \mathrm{CDCl}_{3}\right) \delta 139.11,138.89,138.53,128.76$, 128.68, 128.52, 128.51, 128.12, 128.11, 128.01, 127.74, 127.68, 127.64, 127.18, 82.39, 77.85, 77.08, 75.09, 73.18, 72.84, 70.59, 69.61, 68.27, 51.06, 37.37, 26.94.

\section{(2-azidoethyl)-2-O-[2',3',4',6'-tetra-O-benzoyl- $\alpha$-D mannopyranosyl]-3,4,6-tri-O-benzyl-5a-carba- $\alpha$-D- mannopyranoside (18).}

Trichloroacetimidate $\mathbf{6}^{21}$ (0.208 g, $0.281 \mathrm{mmol}, 1.3$ equiv) and carbamannoside 16 ( $0.112 \mathrm{~g}, 0.216 \mathrm{mmol}, 1.0$ equiv) were dissolved in distilled toluene $(2.0 \mathrm{~mL})$ and dried overnight under vacuum. Acid washed molecular sieves AW 300 were activated under vacuum and added to the reagents. The mixture was dissolved in dry $\mathrm{CH}_{2} \mathrm{Cl}_{2}(2.5$ $\mathrm{mL})$, catalytic amount of TMSOTf (7.8 $\mu \mathrm{L}, 0.0432 \mathrm{mmol}, 0.2$ equiv) was added under nitrogen at $-20^{\circ} \mathrm{C}$ and the reaction mixture was stirred at the same temperature for $3 \mathrm{~h}$. $\mathrm{Et}_{3} \mathrm{~N}(20.0 \mu \mathrm{L})$ was added and the mixture was diluted with $\mathrm{CH}_{2} \mathrm{Cl}_{2}$ and filtered through a Celite ${ }^{\circledR}$ pad. Evaporation of the solvent afforded a crude product (0.290 g), which was subjected to a flash chromatography. Elution with a 7:3 hexane/acetone mixture afforded the $O$-protected pseudomannobioside 18 (0.208 $\mathrm{mg}, 88 \%$ yield), pure as a white solid. m.p. $40-42^{\circ} \mathrm{C} . \mathrm{R} f=0.22$ (7:3 hexane/acetone); $[\alpha]^{20}{ }_{\mathrm{D}}-23.0$ (c 0.63, $\mathrm{CHCl}_{3}$ ). FTIR (neat) v 3350.1, 3294.9, 3069.3, 2961.0, 2875.4, 2106.4, 1724.0, 1601.0, 1450.7, 1259.8, 1094.9, 1065.8, 1026.6, 825.9, $707.9 \mathrm{~cm}^{-1} .{ }^{1} \mathrm{H}$ NMR $(250 \mathrm{MHz}, \mathrm{CDCl} 3) \delta 8.01-8.16(\mathrm{~m}, 5 \mathrm{H}$, Ar), 7.92-8.00 (m, 2H, Ar), 7.70-7.90 (m, 2H, Ar), 7.03-7.69 (m, 26H, Ar), 6.04 (t, J = 9.5 Hz, 1H, H4'), 5.85-5.94 (m, 2H, H2'+H3'), 5.45 (s, 1H, Hanomeric), 4.39-4.92 (m, 9H, benzylic + H5' + H6'a+ H6’b), 4.22 (d, $J=4.2 \mathrm{~Hz}, 1 \mathrm{H}, \mathrm{H} 2), 3.78-3.86$ (m, 2H, H3 + $\mathrm{HCH} 2 \mathrm{CH} 2 \mathrm{~N} 3), \quad 3.68-3.75(\mathrm{~m}, 1 \mathrm{H}, \mathrm{H} 1), 3.49-3.65(\mathrm{~m}, 3 \mathrm{H}$, HCH2CH2N3), 3.30-3.41 (m, 1H, H4), 3.07-3.25 (m, 2H, H6a + H6b), 1.83-2.16 (m, 3H, H5 + H5a + H5a $)$.

${ }^{13} \mathrm{C}$ NMR $\left(\mathrm{CDCl}_{3}\right) \delta 166.31,165.73,165.52,165.24,138.96$, 138.92, 138.59, 133.58, 133.27, 130.01, 129.89, 129.64, 129.37, 129.09, 128.69, 128.61, 128.44, 127.83, 127.64, 127.56, 98.90, 
76.25, 75.11, 73.10, 70.78, 70.40, 70.13, 69.54, 67.99, 67.35, 63.39, $50.91,37.71,27.72$.

\section{(2-aminoethyl)-2-O-[2',3',4',6'-tetra-O-benzoyl- $\alpha$-D-} mannopyranosyl]-5a-carba- $\alpha$-D-mannopyranoside (19).

Pseudomannobioside 18 (0.080 g, $0.073 \mathrm{mmol}, 1.0$ equiv) was dissolved in a 4:1 MeOH/anhydrous DMF mixture $(2.0 \mathrm{~mL})$ and $10 \%$ Pd-C $(0.080 \mathrm{~g})$ was added. The reaction mixture was hydrogenated (1 bar) at room temperature under hydrogen saturated atmosphere until reduction of the azide group and deprotection of the benzyl groups were achieved, monitoring by analytical TLC (8:2 $\left.\mathrm{CH}_{2} \mathrm{Cl}_{2} / \mathrm{MeOH}\right)$. After $18 \mathrm{~h}$ the mixture was diluted with $\mathrm{MeOH}$ and filtered through a Celite ${ }^{\circledR}$ pad. The evaporation of the solvent afforded a crude reaction product $(0.035 \mathrm{~g})$ which was further diluted with $\mathrm{MeOH}$ and filtered off using syringe filter, to remove the residue coal. Evaporation of the solvent afforded a white solid, which was recrystallized from $(i-\mathrm{Pr})_{2} \mathrm{O}$ to yield pure 1,2pseudomannobioside 19 ( $0.031 \mathrm{~g}, 53 \%$ yield), as a yellow solid: $\mathrm{mp}$ : $38-39^{\circ} \mathrm{C} . \mathrm{R} f=0.3\left(8: 2 \mathrm{CH}_{2} \mathrm{Cl}_{2} / \mathrm{MeOH}+0.1 \% \mathrm{Et}_{3} \mathrm{~N}\right) ;[\alpha]^{20}{ }_{\mathrm{D}}-34.85$ (c 0.68, MeOH). FTIR (neat) v 3377.6, 3072.1, 2951.0, 2782.8 1721.2, 1656.4, 1592.8, 1437.7, 1297.2, 1256.9, 1161.3, 1082.4, 1066.3, 1018.5, 857.5, 697.4 $\mathrm{cm}^{-1} .{ }^{1} \mathrm{H}$ NMR (250 MHz, CD $\left.{ }_{3} \mathrm{OD}\right) \delta$ 8.06-8.16 (m, 2H, Ar), 7.88-8.03 (m, 4H, Ar), 7.22-7.80 (m, 14H, Ar), 6.11 (t, $J=9.8 \mathrm{~Hz}, 1 \mathrm{H}, \mathrm{H} 4$ '), 5.89-5.97 (m, 1H, H2'), 5.84 (dd, $J=10.2,3.2 \mathrm{~Hz}, 1 \mathrm{H}, \mathrm{H3}$ '), 5.48 (d, $J=1.4 \mathrm{~Hz}, 1 \mathrm{H}$, Hanomeric), 4.77 (dd, $J=13.4,3.9 \mathrm{~Hz}, 1 \mathrm{H}, \mathrm{H} 5$ '), 4.47-4.58 (m, 2H, H6'a + H6’b), 4.23-4.26 (m, 1H, H2), 3.90-3.97 (m, 1H, H1) 3.45-3.89 (m, $6 \mathrm{H}, \mathrm{H} 3+\mathrm{H} 4+\mathrm{H} 6 \mathrm{a}+\mathrm{H} 6 \mathrm{~b}+\mathrm{OCH} 2), 3.07-3.12(\mathrm{~m}, 2 \mathrm{H}, \underline{\mathrm{CH}} 2 \mathrm{NH} 2)$, 2.00-2.13 (m, 1H, H5), 1.72-1.89 (m, 2H, H5a $\alpha+\mathrm{H} 5 \mathrm{a} \beta) .{ }^{13} \mathrm{C}$ NMR $\left(63 \mathrm{MHz}, \mathrm{CD}_{3} \mathrm{OD}\right) \delta 167.53,167.01,166.79,166.54,134.78$, $134.74,134.57,134.55,131.12,130.76,130.71,130.69,130.60$, $130.51,130.30,130.27,129.84,129.78,129.63,129.47,101.30$, 80.54, 78.24, 74.20, 72.33, 71.71, 71.46, 70.77, 68.31, 66.16, 64.34, 63.92, 40.91, 40.46, 27.79

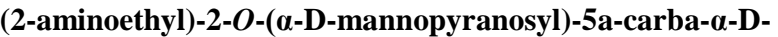 mannopyranoside (5).}

A solution $1 \mathrm{M}$ of MeONa in $\mathrm{MeOH}(65.0 \mu \mathrm{L}, 0.065 \mathrm{mmol}, 1.5$ equiv) was added to a solution of 1,2-pseudomannobioside 19 (0.034 g, $0.043 \mathrm{mmol}, 1.0$ equiv) in $\mathrm{MeOH}(0.8 \mathrm{~mL})$. The reaction mixture was stirred for $12 \mathrm{~h}$ monitoring by TLC $\left(8: 2 \mathrm{CH}_{2} \mathrm{Cl}_{2} / \mathrm{MeOH}\right)$. The reaction mixture was diluted with $\mathrm{MeOH}$ and Amberlite IRC 50 was added until $\mathrm{pH}$ 7. The beads were filtered off and washed with $\mathrm{MeOH}$, then, the filtrated solution was evaporated affording a crude reaction product $(0.015 \mathrm{~g})$. The solid product was dissolved in $\mathrm{MeOH}$ and filtered through a syringe filter. Evaporation of the solvent afforded a yellow solid, which was recrystallized from ( $i$ $\mathrm{Pr})_{2} \mathrm{O}$ to yield fully $\mathrm{O}$-deprotected 1,2-pseudomannobioside 5 (0.011 g, $70 \%$ yield), pure as a pale yellow solid: mp: $103-105{ }^{\circ} \mathrm{C}$. $\mathrm{R} f=$ $0.15\left(1: 1 \mathrm{CH}_{2} \mathrm{Cl}_{2} / \mathrm{MeOH}\right) ;[\alpha]^{20}{ }_{\mathrm{D}}+16.4($ c $1.14, \mathrm{MeOH})$.

FTIR (neat) v 3396.5, 2924.9, 2852.6, 2746.5, 2489.3, 1537.7, 1486.7, 1180.4, 1048.6, $1025.8 \mathrm{~cm}^{-1} .{ }^{1} \mathrm{H}$ NMR $\left(250 \mathrm{MHz}, \mathrm{CD}_{3} \mathrm{OD}\right)$ $\delta 5.06$ (d, $J=1.7 \mathrm{~Hz}, 1 \mathrm{H}$, Hanomeric), 4.03-4.11 (m, $1 \mathrm{H}, \mathrm{CHO}$ ), 3.98 (dd, $J=3.2,1.7 \mathrm{~Hz}, 1 \mathrm{H}, \mathrm{CHO}$ ), 3.42-3.93 (m, 12H, CHO), 3.13 (t, $J=5.1 \mathrm{~Hz}, 2 \mathrm{H}, \underline{\mathrm{CH}} 2 \mathrm{NH} 2), 1.88-1.99$ (m, 1H, H5), 1.53-1.85 (m, $2 \mathrm{H}, \mathrm{H} 5 \mathrm{a} \alpha+\mathrm{H} 5 \mathrm{a} \beta) .{ }^{13} \mathrm{C}$ NMR $\left(63 \mathrm{MHz}, \mathrm{CD}_{3} \mathrm{OD}\right) \delta 104.01,96.65$, 79.60, 78.05, 75.52, 74.19, 72.49, 71.83, 68.75, 66.97, 64.47, 63.19,
41.15, 40.46, 27.74. HRMS: $\left(\mathrm{M}+\mathrm{H}^{+}\right)$found 384.1860; $\mathrm{C}_{15} \mathrm{H}_{30} \mathrm{NO}_{10}$ requires 384.1870 .

\section{3,4,6-tri-O-acetyl-1,2-anhydro-5a-carba- $\beta$-D-mannopyranose (13). ${ }^{25}$}

$\beta$-Epoxide diol 11 (0,060g, $0.24 \mathrm{mmol}, 1.0$ equiv) was dissolved in EtOH $(2.0 \mathrm{~mL})$ and $10 \% \mathrm{Pd}-\mathrm{C}(0.010 \mathrm{~g})$ was added. The reaction mixture was hydrogenated (1 bar) at room temperature under hydrogen saturated atmosphere overnight. The reaction mixture was then diluted with EtOH and filtered through a Celite ${ }^{\circledR}$ pad. The evaporation of the solvent afforded a crude reaction product which was further diluted with EtOH and filtered off using syringe filter, to remove the residue coal. Evaporation of the solvent afforded a crude reaction product $(0.032 \mathrm{~g})$ as a colorless liquid constituted by epoxy triol 12, directly acetylated. Epoxy triol $12(0.032 \mathrm{~g}, 0.20 \mathrm{mmol}, 1.0$ equiv) was dissolved in anhydous pyridine $(0.5 \mathrm{~mL})$ and the reaction mixture was cooled at $0^{\circ} \mathrm{C}$. Acetic anhydride $(0.25 \mathrm{~mL})$ was added and the reaction was stirred at room temperature overnight. Coevaporation of the reaction mixture with toluene afforded a crude product $(0.049 \mathrm{~g})$, which was subjected to flash chromatography. Elution with 1:1 hexane/AcOEt mixture afforded tri-O-acetyl- $\beta$ epoxide $13(0.040 \mathrm{~g}, 70 \%$ yield), pure as a pale yellow oil. $\mathrm{R} f=0.26$ (hexane/AcOEt 1:1); ${ }^{1} \mathrm{H}$ NMR $\left(\mathrm{CDCl}_{3}\right) \delta 5.23(\mathrm{dd}, J=8.7,1.8 \mathrm{~Hz}$, $1 \mathrm{H}, \mathrm{H} 3$ ), 5.07-5.19 (m, 1H, H4), 4.05 (dd, $J=11.4,4.9 \mathrm{~Hz}, 1 \mathrm{H}, \mathrm{H} 6$ ), 3.85 (dd, $J=11.3,3.1 \mathrm{~Hz}, 1 \mathrm{H}, \mathrm{H6}$ '), 3.41 (dd, $J=3.9,1.5 \mathrm{~Hz}, 1 \mathrm{H}$, $\mathrm{H} 1$ ), 3.33 (t, $J=4.2 \mathrm{~Hz}, 1 \mathrm{H}, \mathrm{H} 2$ ), 2.13-2.25 (m, 1H, H5), 2.10 (s, $3 \mathrm{H}, \mathrm{OAc}$ ), 2.04 (s, 3H, OAc), 2.01 (s, 3H, OAc), 1.88-1.97 (m, 2H, $\mathrm{H} 5 \mathrm{a} \alpha+\mathrm{H} 5 \mathrm{a} \beta) .{ }^{13} \mathrm{C}$ NMR $\left(\mathrm{CDCl}_{3}\right) \delta 171.11,170.34,170.02,71.18$, 70.01, 69.90, 63.90, 53.38, 35.72, 29.20, 21.00, 20.96, 20.92.

\section{(2-azidoethyl)-3,4,6-tri-O-acetyl-5a-carba-a-D-mannopyranoside} (15).

Tri-O-acetyl epoxide $13(0.033 \mathrm{~g}, 0.114 \mathrm{mmol}, 1.0$ equiv $)$ was dissolved in a $1.3 \mathrm{M}$ solution of 2-azido-1-ethanol in $\mathrm{CH}_{2} \mathrm{Cl}_{2}{ }^{25}(0.36$ $\mathrm{mL}, 0.456 \mathrm{mmol}, 4.0$ equiv) and $\mathrm{Cu}(\mathrm{OTf})_{2}(0.016 \mathrm{~g}, 0.045 \mathrm{mmol}$, 0.4 equiv) was added. The reaction mixture was stirred for $18 \mathrm{~h}$ at room temperature. Dilution with $\mathrm{CH}_{2} \mathrm{Cl}_{2}$ and evaporation of the washed (1:1 $\mathrm{NaHCO}_{3} / \mathrm{NH}_{4} \mathrm{Cl}$ mixture and brine) and dried $\left(\mathrm{Na}_{2} \mathrm{SO}_{4}\right)$ organic solution afforded a crude reaction product $(0.048$ g) which was subjected to flash chromatography. Elution with a 1:1 hexane/AcOEt solution afforded the glycosyl acceptor 15 (0.035 g, $82 \%$ yield), pure as a pale yellow oil. $\mathrm{R} f=0.14$ (1:1 hexane/AcOEt). $[\alpha]_{\mathrm{D}}^{20}+21.71$ (c 0.35, $\mathrm{CHCl}_{3}$ ). FTIR (neat) v 3480.4, 2956.7, 2925.7, 2855.4, 2106.9, 1738.1, 1454.0, 1368.7, 1227.5, 1098.8, 1039.6, $801.0 \mathrm{~cm}^{-1} .{ }^{1} \mathrm{H}$ NMR $\left(250 \mathrm{MHz}, \mathrm{CDCl}_{3}\right) \delta 5.23-5.34(\mathrm{~m}$, $1 \mathrm{H}, \mathrm{H} 3$ ), 5.20 (dd, $J=9.5,2.7 \mathrm{~Hz}, 1 \mathrm{H}, \mathrm{H} 4$ ), 4.16-4.23 (m, $1 \mathrm{H}, \mathrm{H} 2$ ), 4.11 (dd, $J=11.3,4.9 \mathrm{~Hz}, 1 \mathrm{H}, \mathrm{H6}$ ), 3.94 (dd, $J=11.3,4.9 \mathrm{~Hz}, 1 \mathrm{H}$, H6'), 3.73-3.78 (m, 1H, H1), 3.70 (t, $J=5.0 \mathrm{~Hz}, 2 \mathrm{H}, \mathrm{CH} 2 \mathrm{~N} 3$ ), 3.39 (d, $J=4.6 \mathrm{~Hz}, 1 \mathrm{H}, \mathrm{OCH} 2$ ), 3.35 (d, $J=4.6 \mathrm{~Hz}, 1 \mathrm{H}, \mathrm{OCH} 2$ ), 2.242.36 (m, 1H, H5), 2.08 (s, 3H, OAc), 2.05 (s, 3H, OAc), 2.03 (s, 3H, OAc), 1.86-1.94 (m, 2H, H5a $\alpha+\mathrm{H} 5 \mathrm{a} \beta) .{ }^{13} \mathrm{C}$ NMR $\left(\mathrm{CDCl}_{3}\right) \delta$ 171.13, 170.55, 169.90, 73.87, 69.71, 68.67, 63.93, 50.93, 35.19, 26.77, 21.08, 20.96 . 
(2-azidoethyl)-2-O-[2',3',4',6'-tetra-O-benzoyl- $\alpha$-Dmannopyranosyl]-3,4,6-tri-O-acetyl-5a-carba- $\alpha$-Dmannopyranoside (17).

Trichloroacetimidate $\mathbf{6}^{21}(0.047 \mathrm{~g}, 0.064 \mathrm{mmol}, 1.3$ equiv) and carbamannoside 15 (0.019 g, $0.049 \mathrm{mmol}, 1.0$ equiv) were dissolved in distilled toluene $(2.0 \mathrm{~mL})$ and dried overnight under vacuum.

Acid washed molecular sieves AW 300 were activated under vacuum and added to the reagents. The mixture was dissolved in dry $\mathrm{CH}_{2} \mathrm{Cl}_{2}(1.0 \mathrm{~mL})$ and a catalytic amount of TMSOTf $(1.76 \mu \mathrm{L}$, $0.010 \mathrm{mmol}, 0.2$ equiv) was added under nitrogen at $-20^{\circ} \mathrm{C}$ and the reaction mixture was stirred at the same temperature for 20 minutes, monitoring by TLC (1:1 hexane/AcOEt $). \mathrm{Et}_{3} \mathrm{~N}(10.0 \mu \mathrm{L})$ was added and the mixture was diluted with $\mathrm{CH}_{2} \mathrm{Cl}_{2}$ and filtered through a Celite ${ }^{\circledR}$ pad. Evaporation of the solvent afforded a crude product (0.034 g), which was subjected to a flash chromatography. Elution with a 1:1 hexane/AcOEt mixture as eluent afforded the $O$-protected pseudomannobioside 17 (0.025 g, 53\% yield) pure as a pale yellow oil. $\mathrm{R} f=0.40$ (1:1 hexane/AcOEt). $[\alpha]^{20}{ }_{\mathrm{D}}-42.8\left(\right.$ (c 1.7, $\left.\mathrm{CHCl}_{3}\right)$.

${ }^{1} \mathrm{H}$ NMR $\left(\mathrm{CDCl}_{3}\right) \delta$ 7.94-8.15 (m, 6H, Ar), 7.79-7.89 (m, 2H, Ar), 7.21-7.67 (m, 12H, Ar), 6.06 (t, 1H, $J=9.9$ Hz, H4'), 5.92 (dd, 1H, $J$ = 10.2, $3.1 \mathrm{~Hz}, \mathrm{H} 2$ '), 5.69 (dd, 1H, $J=2.9,1.6 \mathrm{~Hz}, \mathrm{H3}$ '), 5.36 (d, $1 \mathrm{H}, J=10.1 \mathrm{~Hz}, \mathrm{H} 3$ ), 5.32 (d, 1H, $J=1.6 \mathrm{~Hz}$, Hanomeric), 5.26 (dd, 1H, $J=9.8,2.8 \mathrm{~Hz}, \mathrm{H} 4$ ), 4.69 (dd, 1H, $J=12.0,2.5 \mathrm{~Hz}, \mathrm{H6}$ 'a), 4.50 (dd, $1 \mathrm{H}, J=12.0,5.4 \mathrm{~Hz}, \mathrm{H6}$ 'b), 4.36-4.45 (m, 1H, H5'), 4.31 (t, $1 \mathrm{H}, J=3.2 \mathrm{~Hz}, \mathrm{H} 2$ ), 4.17 (dd, $1 \mathrm{H}, J=11.4,5.7 \mathrm{~Hz}, \mathrm{H} 6 \mathrm{a}$ ), 4.03 (dd, $1 \mathrm{H}, J=11.4,3.6 \mathrm{~Hz}, \mathrm{H} 6 \mathrm{~b}), 3.73-3.80$ (m, 1H, H1), 3.44-3.60 (m, 2H, CH2N3), 3.13-3.36 (m, 2H, OCH2), 2.21-2.45 (m, 1H, H5), 2.16 (s, 3H, OAc), 2.13 (s, 3H, OAc), 2.04 (s, 3H, OAc), 2.00 (dd, $2 \mathrm{H}, J=8.4,2.3 \mathrm{~Hz}, \mathrm{H} 5 \mathrm{a} \alpha+\mathrm{H} 5 \mathrm{a} \beta) .{ }^{13} \mathrm{C} \mathrm{NMR}\left(\mathrm{CDCl}_{3}\right) \delta 171.37$, $170.67,170.06,166.24,165.81,165.64,165.31,133.69,133.39$, $130.00,129.86,129.43,129.19,128.98,128.78,128.63,128.46$, 99.03, 76.32, 76.14, 72.44, 70.84, 69.96, 69.73, 69.26, 68.58, 67.45, 63.85, 63.41, 50.80, 35.40, 20.94.

\section{(2-azidoethyl)-2-O-( $\alpha$-D-mannopyranosyl)-5a-carba- $\alpha$-D-}

\section{mannopyranoside (4).}

Compound 17 (0.025 g, $0.026 \mathrm{mmol}, 1.0$ equiv) was dissolved in $\mathrm{MeOH}(0.8 \mathrm{~mL})$ and cooled at $0{ }^{\circ} \mathrm{C}$. A freshly prepared $1.0 \mathrm{M}$ solution of $\mathrm{MeONa} / \mathrm{MeOH}$ (78 $\mu \mathrm{L}, 0.078 \mathrm{mmol}, 0.3$ equiv) was added to the resulting solution and the reaction mixture was stirred for $12 \mathrm{~h}$ at room temperature. The mixture was then neutralized with an acidic Amberlite ${ }^{\mathrm{TM}}$ IR $120 \mathrm{H}$ resin The resin was then removed by filtration and repeatedly extracted with methanol. The combined filtrate was concentrated under vacuum to give a crude product, which was recrystallized from $i$-PrOH to yield pure glycoside 4 (0.010 g, yield $88 \%)$ as a white solid: $\mathrm{mp}: 85-88^{\circ} \mathrm{C}$. $\mathrm{R} f=0.21(1: 1$ $\left.\mathrm{CH}_{2} \mathrm{Cl}_{2} / \mathrm{MeOH}\right) .[\alpha]_{20}{ }^{\mathrm{D}}-43.2(c \quad 0.36, \mathrm{MeOH}){ }^{1} \mathrm{H}$ NMR $\left(\mathrm{D}_{2} \mathrm{O}\right) \delta$ 4.98 (s, 1H, Hanomeric), 4.04 (bs, 2H, H1 + H2), 3.95 - 3.50 (m, $12 \mathrm{H}, \mathrm{CHO}, \mathrm{CH} 2 \mathrm{O}, \mathrm{CH} 2 \mathrm{~N} 3), 3.44-3.37$ (m, 2H, OCH2), $1.96-$ $1.86(\mathrm{~m}, 1 \mathrm{H}, \mathrm{H} 5), 1.80-1.54(\mathrm{~m}, 2 \mathrm{H}, \mathrm{H} 5 \mathrm{a} \alpha+\mathrm{H} 5 \mathrm{a} \beta) .{ }^{13} \mathrm{C} \mathrm{NMR}$ $\left(\mathrm{D}_{2} \mathrm{O}, 0.1 \% \mathrm{CD}_{3} \mathrm{CN}\right) \delta 101.70,78.45,75.15,72.81,71.49,69.51$, 69.23, 69.09, 66.77, 65.93, 61.30, 60.20, 49.57, 37.82, 25.40. HRMS: $(\mathrm{M}+\mathrm{Na}+)$ found 432.1586; $\mathrm{C}_{15} \mathrm{H}_{27} \mathrm{~N}_{3} \mathrm{O}_{10} \mathrm{Na}$ requires 432.1594 .

\section{Biological assays}

Surface plasmon resonance analysis. DC-SIGN ECD production and purification: DC-SIGN extracellular domain (DC-SIGN ECD) construct was produced and purified as described previously. ${ }^{31}$

Surface Plasmon Resonance analysis: Surface plasmon resonance experiments were performed on a Biacore 3000 using a CM4 chip, functionalized at $5 \mu \mathrm{L} / \mathrm{min}$. BSA or BSAMan were immobilized on flow cells using amine-coupling method. Fc1 was prepared as reference surface. Flow cell (Fc) 1 and 2 were activated with $50 \mu \mathrm{L}$ of a $0.2 \mathrm{M}$ EDC/ $0.05 \mathrm{M}$ NHS mixture. After this step, Fc1 and Fc2 were respectively functionalized with bovine serum albumine (BSA) and mannosylated bovine serum albumine (BSA-Mannotriose, BSA-man 1 1-3[man 1 1-6]man, 12 glycosylated sites, , 60 $\mu$ g.mL-1, Dextra laboratories). Then remaining activated groups of both cells were blocked with $30 \mu \mathrm{L}$ of $1 \mathrm{M}$ ethanolamine. After blocking, the two Fc were treated with 5 $\mu \mathrm{L}$ of $10 \mathrm{mM} \mathrm{HCl}$ to remove unspecific bound protein and $5 \mu \mathrm{L}$ of $50 \mathrm{mM}$ EDTA to expose surface to regeneration protocol. Finally, 2381 RU and 1847 RU of BSA and BSA-Man were respectively immobilized on Fc1 and Fc2. The selected concentration was $20 \mu \mathrm{M}$ for DC SIGN ECD.

For inhibition studies, 20 (M of DC-SIGN ECD mixed with increasing concentrations of inhibiting compounds were prepared in a running buffer composed of $25 \mathrm{mM}$ Tris $\mathrm{pH} 8$, 150 mM NaCl, 4 mM CaCl2, 0.005\% P20 surfactant, and 13 / of each sample was injected onto the surfaces at a $5 \mu \mathrm{L} / \mathrm{min}$ flow rate. The resulting sensorgrams were reference surface corrected.

The DC-SIGN binding responses were extracted from sensorgrams, converted to percent residual activity values $(y)$ with respect to lectin alone binding, and plotted against corresponding compound concentration. The 4-parameter logistic model (equation 1) was fitted to the plots, and the $\mathrm{IC}_{50}$ values were calculated, from equation 2, using the values of fitted parameters (Rhi, Rlo, $A 1$ and $A 2$ ).

$$
y=R_{h i}-\frac{R_{h i}-R_{l o}}{1+\left(\frac{\text { Conc }}{A_{1}}\right)^{A_{2}}} \quad I C_{50}=A_{1} \cdot\left(\left(\frac{R_{h i}-R_{l o}}{R_{h i}-50}\right)^{\frac{1}{A_{2}}}-1\right)
$$

Figure 4 Equation for percent residual activity values and for IC50 values

\section{Acknowledgements}

This work used the platforms of the Grenoble Instruct centre (ISBG ; UMS 3518 CNRS-CEA-UJF-EMBL), notably the MP3 and SPR platforms, with support from FRISBI (ANR-10-INSB05-02) and GRAL (ANR-10-LABX-49-01) within the Grenoble Partnership for Structural Biology (PSB). V.Porkolab was supported by a grant from la Région Rhône-Alpes. F.Fieschi and A.Bernardi acknowledge for support from CM1102 COST Action. V. Di Bussolo acknowledges the University of Pisa (Progetti di Ricerca di Ateneo, PRA 2015 0015) for funding. 


\section{Notes and references}

${ }^{a}$ Dipartimento di Farmacia, Università di Pisa, Via Bonanno 33, 56126 Pisa, Italy.

${ }^{b}$ Univ. Grenoble Alpes, CNRS, CEA, Institut de Biologie Structurale, F38044 Grenoble, France.

${ }^{c}$ Dipartimento di Chimica, Università degli Studi di Milano, via Golgi 19, 20133 Milano, Italy

${ }^{d}$ Dipartimento di Chimica e Chimica Industriale, Università di Pisa, Via Moruzzi 13, 56125 Pisa, Italy; E-mail: valeria.dibussolo@unipi.it.

$\dagger \quad$ Electronic Supplementary Information (ESI) available: [NMR spectra of 4 and 5, 10-18; HRMS of 4 and 5; sensorgrams and inhibition curves of $\mathbf{4 , 5}$ and of natural epitope Man〈(1,2)Man]. See DOI:

1. A. Imberty, A. Varrot, Curr Opin Struct Biol, 2008, 18, 567-576.

2. R. A. Dwek, Chem. Rev. 1996, 96, 683-720.

3. L. L. Kiessling, J.C. Grim, Chem. Soc. Rew. 2013, 42, 4476-4491.

4. S. Cecioni, A. Imberty, S. Vidal, Chem.Rew. 2015, 115, 525-561.

5. T. B. H. Geijtenbeek, R. Torensma, S. J. van Vliet, G. C. F. van Duijnhoven, G. J. Adema, Y. van Kooyk, G.C. Figdor, Cell, 2000, 100, 575-585.

6. S. K. Wang, P. H. Liang, R.D. Astronomo, T. L. Hsu, S.-L. Hsieh, D. R. Burton, C. H. Wong, PNAS, 2008, 105, 3690-3695.

7. M. J., Borrok, L. L. Kiessling, J.Am.Chem.Soc. 2007, 129, 1278012785.

8. K. C. A. Garber, K. Wangkanont, E. E. Carlson, L. L. Kiessling, Chem.Commun. 2010, 46, 6747-6749.

9. S. L. Mangold, L. R. Prost, L. L. Kiessling, Chem. Sci. 2012, 3, 772777.

10. J. J. Reina, S. Sattin, D. Invernizzi, S. Mari, L. Martinez-Prats, G. Tabarani, F. Fieschi, R. Delgado, P. M. Nieto, J. Rojo, A. Bernardi,. ChemMedChem 2007, 2(7), 1030-1036.

11. G. Timpano, G. Tabarani, M. Anderluh, D. Invernizzi, F.Vasile, D. Potenza, P.M. Nieto, J. Rojo, F. Fieschi, A. Bernardi, ChemBioChem 2008, 9(12), 1921-1930.

12. S. Sattin, A. Daghetti, M. Thepaut, A. Berzi, M. Sanchez-Navarro, G. Tabarani, J. Rojo, F. Fieschi, M. Clerici, A. Bernardi, ACS Chem. Biol. 2010, 5(3), 301-312.

13. M. Andreini, D. Doknic, I. Sutkeviciute, J.J. Reina, J. Duan, E. Chabrol, M. Thepaut, E. Moroni, F. Doro, L. Belvisi, J. Weiser, J. Rojo, F. Fieschi, A. Bernardi, Org. Biomol. Chem. 2011, 9(16), 5778-5786.

14. J. Luczkowiak, S. Sattin, I. Sutkeviciute, J.J. Reina, M. SanchezNavarro, M. Thepaut, L. Martinez-Prats, A. Daghetti, F. Fieschi, R. Delgado, A. Bernardi, J. Rojo, Bioconjugate Chem. 2011, 22(7), 1354-1365.

15. A. Berzi, J. J. Reina, R. Ottria, I. Sutkeviciute, P. Antonazzo, M. Sanchez-Navarro, E. Chabrol, M. Biasin, D. Trabattoni, I. Cetin, J. Rojo, F. Fieschi, A. Bernardi, M. Clerici, AIDS 2012, 26(2), 127-137.

16. N. Obermajer, S. Sattin, C. Colombo, M. Bruno, U. Svajger, M. Anderluh, A. Bernardi, Molecules Molecular Diversity 2011, 15(2), 347-360.
17. N. Varga, I. Sutkeviciute, C. Guzzi, J. McGeagh, I. Petit-Haertlein, S. Gugliotta, J. Weiser, J. Angulo, F. Fieschi, A. Bernardi, Chem. Eur J. 2013, 19(15), 4786-4797.

18. O. Martinez-Avila, K. Hijazi, M. Marradi, C. Clavel, C. Campion, C. Kelly, C.; Penades, S. Chem. Eur. J. 2009, 15(38), 9874-9888.

19. M. Thepaut, C. Guzzi, I. Sutkeviciute, S. Sattin, R. Ribeiro-Viana, N. Varga, E. Chabrol, J. Rojo, A. Bernardi, J. Angulo, P. M. Nieto, F. Fieschi, J. Am. Chem. Soc. 2013, 135(7), 2518-2529.

20. S. Mari, H. Posteri, G. Marcou, D. Potenza, F. Micheli, F. J. Canada, J. Jimenez-Barbero, A. Bernardi, Eur. J. Org. Chem. 2004, 24, 51195125.

21. D. J. Lee, R. Kowalczyk, V. J. Muir, P. M. Rendle, M. A. Brimble, Carbohydr. Res. 2007, 342(17), 2628-2634.

22. I. Frau, V. Di Bussolo, L. Favero, M. Pineschi, P. Crotti, Chirality 2011, 23(9), 820-826.

23. A. H. Hoveyda, D. A. Evans, G. C. Fu, Chem. Rev. 1993, 93(4), 1307-70.

24. S. Ogawa, T. Tonegawa, Carbohydr. Res. 1990, 204, 51-64.

25. V. W.-F. Tai, P. -H. Fung, Y.-S. Wong, T. K. M. Shing, Tetrahedron:Asymmetry 1994, 5(7), 1353-1362.

26. Y. Dong, X. Liang, H. Yuan, S. Qi, F. Chen, D. Wang, Green Chem. 2008, 10(9), 990-994.

27. A. Bernardi, D. Arosio, L. Manzoni, F. Micheli, A. Pasquarello, P. Seneci, J. Org. Chem. 2001, 66(19), 6209-6216.

28. S. Ogawa, S. Sasaki, H. Tsunoda, Chem. Lett. 1993, 9, 1587-1590.

29. J. Frigell, I. Cumpstey, Beilstein J. Org. Chem. 2010, 6, 1127-1131.

30. G. Timpanaro, G. Tabarani, M. Anderluh, D. Invernizzi, F. Vasile, D. Potenza, P. M. Nieto, J. Rojo, F. Fieschi, A. Bernardi, ChemBioChem 2008, 9, 1921-1930.

31. G. Tabarani, M. Thépaut, D. Stroebel, C. Ebel, C. Vivès, P. Vachette, D. Durand, F. Fieschi, 2009, 284(32), 21229-21240. 